\title{
The Characteristic Analysis of Web User Clusters Based on Frequent Browsing Patterns
}

\author{
Zhiwang Zhang and Yong Shi \\ School of Information of Graduate University of Chinese Academy of Sciences, Chinese \\ Academy of Sciences Research Center on Data Technology and Knowledge Economy, Beijing \\ (100080), China \\ zzwmis@sohu.com \\ Chinese Academy of Sciences Research Center on Data Technology and Knowledge Economy, \\ Graduate University of Chinese Academy of Sciences, Beijing (100080), China \\ yshi@gucas.ac.cn

\begin{abstract}
Web usage mining (WUM) is an important and fast developing area of web mining. Recently, some enterprises have been aware of its potentials, especially for applications in Business Intelligence (BI) and Customer Relationship Management (CRM). Therefore, it is crucial to analyze the behaviors and characteristics of web user so as to use this knowledge for advertising, targeted marketing, increasing competition ability, etc. This paper provides an analytic method, algorithm and procedure based on suggestions from literature and the authors' experiences from some practical web mining projects. Its application shows combined use of frequent sequence patterns (FSP) discovery and the characteristic analysis of user clusters can contribute to improve and optimize marketing and CRM.
\end{abstract}

Keywords: WUM, FSP, clustering.

\section{Introduction}

Data mining has been used by many organizations to extract the valuable information from large volumes of data and then use them to make critical business decisions. As for WUM, a lot of work mainly focus on web user navigating patterns discovery and association analysis, user and web pages clustering. However, it is insufficient in analyzing the characteristics of web clusters after identifying interesting frequent browsing patterns. In this paper, firstly we introduce related work in WUM and its analytic steps. And then, we discuss the three main steps in WUM, taking an educational web server for example. Here our main work lies in creating a data mart of web usage data, discovering some FSP of web user, providing a method that measures the similarities among different FSP for user clusters, and providing an algorithm and its applications. In the end, a comparison between this algorithm and kmeans, Kohonen is given. 


\section{Related Work}

\subsection{Taxonomy of Web Mining}

Web mining involves a wide range of applications that aims at discovering and extracting hidden information in data stored on the Web. Besieds, Web mining can be categorized into three different classes: (i) Web content mining, (ii) Web structure mining and (iii) WUM. For detailed surveys of Web mining please refer to [1]. Respectively, Web content mining [1] is the task of discovering useful information available on-line. Web structure mining $[1,2,3]$ is the process of discovering the structure of hyperlinks within the Web. WUM is the task of discovering the activities of the users while they are browsing and navigating through the Web [1].

\subsection{WUM}

WUM, from the data mining aspect, is the task of applying data mining techniques to discover usage patterns from Web data in order to understand and better serve the needs of users navigating on the Web [1]. As every data mining task, the process of WUM also consists of three main steps: (i) data selecting and preprocessing, (ii) pattern discovery and (iii) pattern analysis.

\section{Data Selecting and Preprocessing}

After we set up a definite business target, it is necessary to extract and select the types of data from data sources. In general, in WUM we may gain the following three types of data: (i) web data: generated by visits to a web site; (ii) business data: produced by the respective OLTP, OLAP, DSS and other systems; (iii) meta data, data describing the web site itself, for example, content and structure, etc.

Just as the discussion above, Web log data can mainly get from the internet or intranet resources. In this paper, web log files are from DePaul CTI Web server log. The preprocessed and filtered data is based on a random sample of users visiting this site for a 2 week period which contains 13745 sessions and 683 page views from 5446 users. To analyze, we have developed a data mart to support FSP mining and further the characteristics analysis of user clusters. The data model is reported in Figure1.

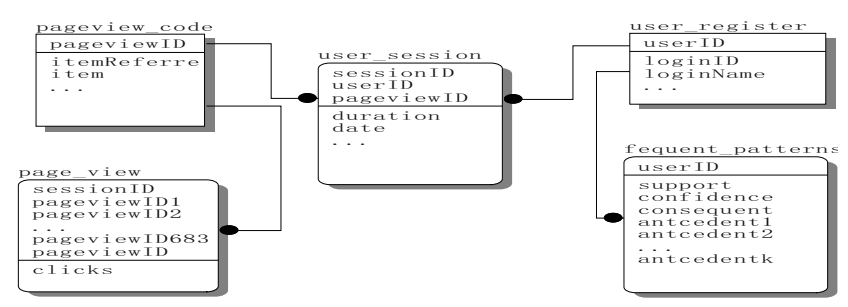

Fig. 1. Data mart tables and their relations 


\section{Frequent Patterns Discovery}

\subsection{FSP Discovery}

In this section, we use the notion of FSP discovery where temporal correlations among items in user transactions are discovered.

\subsection{Results of Experimentation}

In this paper we employ FSP discovery to the above dataset and produce these results in Fig. 2 with maximum length be 3 and the top 3 of these FSP numbered 3514.

\section{User Support Confidence Antecedent $1==>$ Antecedent $2==>$ Consequent}

$4 \quad 0.0204 \quad 1.0000 \quad$ /programs/2002/bachelorcs2002.asp ==>/news/default.asp

$\begin{array}{llll}5 & 0.0255 & 1.0000 \quad \text { /admissions/international.asp }==>\text { /admissions/ }\end{array}$

$4 \quad 0.0204 \quad 1.0000 \quad$ /authenticate/login.asp?section=advising\&title =appointment\&urlahead=apt_making/makeapts ==>/news/default.asp

$\begin{array}{llll}7 & 0.0357 & 1.0000 \quad / \mathrm{cti} / \text { core/core.asp?section=news }==>/ \text { news } / \text { default.asp }\end{array}$

$40.02041 .0000 \quad$ /news/news.asp?theid=586 ==>/news/default.asp

Fig. 2. FSP on Web server log

\section{The Characteristics Analysis of Web User Clusters}

\subsection{Similarity Measures}

Given a weighted value set $W$ of the pages in the $k-t h$ length FSP $f p_{i}$, and thus, the similarity between the FSP $f p_{i}$ and $f p_{j}$ is defined as:

$$
\operatorname{sim}\left(f p_{i}, f p_{j}\right)=\sum_{l=1}^{k} w_{l} * e q\left(p_{i l}, p_{j l}\right), e q\left(p_{i l}, p_{j l}\right)=\left\{\begin{array}{l}
1 \text { if the two pages is same } \\
0 \text { otherwise }
\end{array} .\right.
$$

Here $e q(*, *)$ may be the combined probability distribution function of the $f p_{i}$ and $f p_{j}$.

\subsection{Algorithm}

In this part, we give an algorithm which implement maximum spanning tree (MST) clustering based on the above FSP (MSTCBFSP), as the following:

Input: $F P=\left\{f p_{1}, f p_{2}, \cdots, f p_{m}\right\}, W=\left\{w_{1}, w_{2}, \cdots, w_{k}\right\}, \gamma$. Output: $\mathrm{T} / /$ Set of clusters. Processing Flow: // MST clustering based on FSP.

Step one: Compute the similarity of the different FSP and construct a graph $G$.

Step two: Building a MST $T_{\max }$ on graph $G$.

Step three: Clustering analysis according to the MST $T_{\max }$. 


\subsection{Results of Experimentation}

Each gets the five clusters (Table1.) of web user with the different characteristics after we run algorithm MSTCBFSP, k-means and Kohonen on the above data set of FSP.

Table 1. The comparison of the results between MSTBFSP and k-means, Kohonen

\begin{tabular}{ccrccc}
\hline Algorithm & Clusters & $\begin{array}{c}\text { Percent } \\
\text { (User) }\end{array}$ & $\begin{array}{c}\text { Antecedent1(Pagevie } \\
\text { wID, UserPercent) }\end{array}$ & $\begin{array}{c}\text { Antecedent2(Pagevie } \\
\text { wID, UserPercent) }\end{array}$ & $\begin{array}{c}\text { Consequent(Pagevi } \\
\text { ewID, UserPercent) }\end{array}$ \\
\hline K-means & Cluster\#1 & $3.59 \%$ & $359100 \% 5570 \%$ & $67100 \% 10 \%$ & $388100 \% 6660 \%$ \\
& Cluster\#2 & $64.54 \%$ & $557100 \% 3590 \%$ & $1100 \% 670 \%$ & $3880 \% 660 \%$ \\
& Cluster\#3 & $24.70 \%$ & $557100 \% 3590 \%$ & $67100 \% 10 \%$ & $388100 \% 6660 \%$ \\
& Cluster\#4 & $6.77 \%$ & $359100 \% 5570 \%$ & $10 \% 670 \%$ & $3880 \% 660 \%$ \\
& Cluster\#5 & $0.40 \%$ & $3590 \% \quad 5570 \%$ & $10 \% 670 \%$ & $3880 \% 666100 \%$ \\
\hline \multirow{2}{*}{ Kohonen } & Cluster-1 & $23.90 \%$ & $557100 \% 3590 \%$ & $10 \% 670 \%$ & $388100 \% 6660 \%$ \\
& Cluster-2 & $64.94 \%$ & $557100 \% 3590 \%$ & $1100 \% 670 \%$ & $3880 \% 666100 \%$ \\
& Cluster-3 & $0.80 \%$ & $3590 \% 5570 \%$ & $10 \% 67100 \%$ & $388100 \% 6660 \%$ \\
& Cluster-4 & $3.59 \%$ & $359100 \% 5570 \%$ & $10 \% 67100 \%$ & $388100 \% 6660 \%$ \\
& Cluster-5 & $6.77 \%$ & $359100 \% 5570 \%$ & $10 \% 670 \%$ & $3880 \% 660 \%$ \\
\hline MSTCBFSP & Cluster+1 & $65.74 \%$ & $3590 \% 557100 \%$ & $670 \% 1100 \%$ & $3880 \% 666100 \%$ \\
& Cluster+2 & $22.71 \%$ & $557100 \% 3590 \%$ & $10 \% 67100 \%$ & $388100 \% 6660 \%$ \\
& Cluster+3 & $2.39 \%$ & $5570 \% 359100 \%$ & $67100 \% 10 \%$ & $388100 \% 6660 \%$ \\
& Cluster+4 & $0.40 \%$ & $3590 \% \quad 5570 \%$ & $10 \% 670 \%$ & $3880 \% 666100 \%$ \\
& Cluster+5 & $8.76 \%$ & $359100 \% 5570 \%$ & $10 \% 670 \%$ & $3880 \% 660 \%$ \\
\hline
\end{tabular}

\section{Comparison of the Results and Conclusions}

We use the MSTCBFSP could promptly find a global optimum solution and randomshaped clusters. In contrast, k-means is apt to find a local optimum solution and it does not work on categorical data directly and can only find convex-shaped clusters. Besides, for Kohonen map, the explanation of clustering results is very difficult. In conclusion, the MSTCBFSP is a better method. Consequently, we may try to use the fuzzy MSTCBFSP in the future.

Acknowledgements. This research has been partially supported by a grant from National Natural Science Foundation of China (\#70621001, \#70531040, \#70501030, \#70472074, \#9073020), 973 Project \#2004CB720103, Ministry of Science and Technology, China, and BHP Billiton Co., Australia.

\section{References}

1. Margaret H. Dunham, Data Mining Introductory and Advanced topics, Prentice Hall (2003) 206-218.

2. Ajith Abraham, Business Intelligence from Web Usage Mining, Journal of Information \& Knowledge Management, Vol. 2, No. 4 (2003) 375-390. 